\title{
Nicotine promotes the development of oral leukoplakia via regulating peroxiredoxin 1 and its binding proteins
}

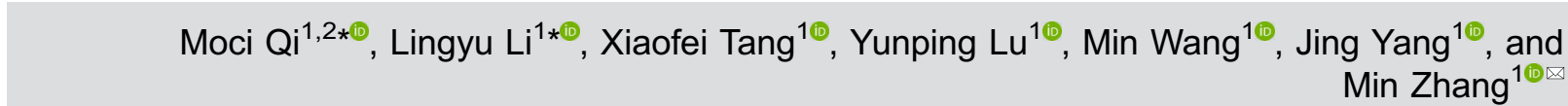 \\ ${ }^{1}$ Beijing Institute of Dental Research, Beijing Key Laboratory, Beijing Stomatological Hospital and School of Stomatology, Capital \\ Medical University, Dongcheng District, Beijing, China \\ ${ }^{2}$ Department of Clinical Laboratory Medicine, Beijing Shijitan Hospital, Capital Medical University, Haidian District, Beijing, China
}

\begin{abstract}
Tobacco can induce reactive oxygen species (ROS) production extensively in cells, which is a major risk factor for oral leukoplakia (OLK) development. Peroxiredoxin 1 (Prx1) is a key antioxidant protein, upregulated in a variety of malignant tumors. We previously found that nicotine, the main ingredient of tobacco, promotes oral carcinogenesis via regulating Prx1. The aim of the present study was to screen and identify the Prx1 interacting proteins and investigate the mechanisms of nicotine on the development of OLK. Through liquid chromatography-tandem mass spectrometry combined with bioinformatics analysis, the candidate Prx1 interacting proteins of cofilin-1 (CFL1), tropomyosin alpha-3 chain (TPM3), and serine/threonine-protein phosphatase 2A $65 \mathrm{kDa}$ regulatory subunit A alpha isoform (PPP2R1A) were screened in human dysplastic oral keratinocyte cells treated with nicotine. CFL1, TPM3, and PPP2R1A were highly expressed in human OLK tissues. The expression of CFL1 increased and the expression of PPP2R1A decreased in OLK of smokers compared to that in OLK of non-smokers. Nicotine upregulated CFL1 and downregulated PPP2R1A in 4-nitro-quinoline-1-oxide (4NQO)-induced OLK tissues in mice in part dependent on Prx1. Furthermore, the in-situ interaction of CFL1, TPM3, and PPP2R1A with Prx1 were validated in human OLK tissues. Our results suggested that tobacco might promote the development of OLK via regulating Prx1 and its interacting proteins CFL1 and PPP2R1A.
\end{abstract}

Key words: Peroxiredoxin 1; Oral leukoplakia; Nicotine; Binding protein; Cell proliferation

\section{Introduction}

Tobacco is the major risk factor associated with oral leukoplakia (OLK) malignant transformation, which has an effect of promoting generation of reactive oxygen species (ROS) and causing oxidative damage to the oral mucosa $(1,2)$. As the main addictive component of tobacco, nicotine affects tumorigenesis by regulation of cell survival, apoptosis, proliferation, metastasis, angiogenesis, and immunosurveillance (3). Peroxiredoxin 1 (Prx1) is an extensively distributed cellular antioxidant, which plays an important role in maintaining the intracellular redox homeostasis. Prx1 can remove excess ROS or act as a molecular chaperone delivering peroxide signals when ROS level is abnormally elevated $(4,5)$. Its high abundance in various tumor tissues suggests that Prx1 is closely related with the development of tumors (6). In a previous study, we found that Prx1 upregulated significantly in OLK and oral squamous cell carcinoma (OSCC) tissues and the expression of Prx1 in OLK and OSCC tissues of smokers was significantly higher than that in non-smokers. Additionally, nicotine upregulated the expression of Prx1 in oral precancerous lesion dysplastic oral keratinocyte (DOK) and OSCC cells and promoted the development of oral precancerous lesions by regulating Prx1 expression involved in cell proliferation and apoptosis $(7,8)$. These results suggest that tobacco might promote the development of OLK by regulating Prx1. However, extensive research is necessary to elucidate the molecular function of Prx1 in tobacco-related OLK.

In the present study, we screened and validated the Prx1 binding proteins of cofilin-1 (CFL1), tropomyosin alpha-3 chain (TPM3), and serine/threonine-protein phosphatase $2 \mathrm{~A} 65 \mathrm{kDa}$ regulatory subunit $A$ alpha isoform

Correspondence: Min Zhang: <zhangminkqyy@hotmail.com>

${ }^{*}$ These authors contributed equally to this work. 
(PPP2R1A), and examined the protein expression in both OLK tissues in the Prx $1^{+/+}$and Prx $1^{+/-}$mouse model and human OLK tissues to further investigate the underlying mechanisms for tobacco promoting the development of OLK by regulating Prx1. Our study revealed some new molecular mechanisms of OLK pathogenesis.

\section{Material and Methods}

\section{Cell culture and treatment}

Human DOK cells (provided by Professor Xiaoxin Chen from North Carolina Central University, USA) were maintained in high-glucose Dulbecco's modified Eagle's medium (DMEM) supplemented with $10 \%(\mathrm{v} / \mathrm{v})$ fetal bovine serum (FBS) (Gibco, USA), containing 100 units/ $\mathrm{mL}$ penicillin and $100 \mu \mathrm{g} / \mathrm{mL}$ streptomycin. DOK cells were treated with $1 \mu \mathrm{M} / \mathrm{L}$ of nicotine (Sigma-Aldrich, USA) for 7 days in a $5 \% \mathrm{CO}_{2}$ atmosphere at $37^{\circ} \mathrm{C}$.

\section{LC-MS/MS and bioinformatics analysis}

The total protein of the nicotine treatment group and the control group was extracted and incubated with anti-Prx1 antibody (1:1000, Abcam, USA) for Co-IP assay to enrich Prx1 interacting proteins. The peptides were analyzed by liquid chromatography-tandem mass spectrometry (LC-MS/MS), and Mascot (http://www.matrix science.com/) and UniProt (https://www.uniprot.org/) databases were used to identify proteins from the peptide sequences. The proteins with unique peptide counts $\geqslant 1$ were considered receivable. Gene ontology (GO), Kyoto Encyclopedia of Genes and Genomes (KEGG) pathway, and protein-protein interaction (PPI) analysis were performed. Prx1 interacting proteins related to cell proliferation were screened based on bioinformatics analysis results.

\section{Animal assay}

An animal model of 4-nitro-quinoline-1-oxide (4NQO)induced OLK established in the tongue tissues of wildtype $\left(\operatorname{Prx} 1^{+/+}\right)$and Prx1 knockdown $\left(\operatorname{Prx} 1^{+/-}\right)$mice (9) was used in the study. Wild type C57BL/6 mice were purchased from Vital River Laboratory Animal Technology (China). One hundred and forty 6-8-week-old C57BL/6 mice $(250-300 \mathrm{~g}$ ) were maintained under standard conditions in accordance with institutional guidelines. The experimental protocol was approved by the Ethics Committee of Capital Medical University (approval No. KQYY-201503-010, protocol \# NSFC 81470752).

Both Prx1 $1^{+/+}$and Prx1 ${ }^{+/-}$mice were randomly divided into four groups: control $(n=10)$, treated with nicotine $(n=20)$, treated with $4 \mathrm{NQO}(\mathrm{n}=20)$, and treated with $4 \mathrm{NQO}+$ nicotine $(n=20)$. The control group received vehicle (distilled water) treatment. 4NQO group was treated with $4 \mathrm{NQO}$ solution $(50 \mu \mathrm{g} / \mathrm{mL})$ in drinking water. The nicotine group received $5 \%$ nicotine treatment on the tongue mucosa 3 times a week. The $4 \mathrm{NQO}+$ nicotine group received both $4 \mathrm{NQO}$ and nicotine treatment as above. At the end of treatment (16 weeks), the tongues of all mice were removed after the mice were sacrificed. Histological observation and pathological diagnosis results showed that the oral precancerous lesions were induced successfully by $4 \mathrm{NQO}$ on the tongue mucosa of Prx1 $1^{+/+}$ and $\operatorname{Prx} 1^{+l-}$ mice.

\section{Patients and tissue specimens}

Twenty-five OLK and ten normal oral mucosa samples were obtained from patients who underwent treatment at Beijing Stomatological Hospital, Capital Medical University during 2015-2017. The OLK specimens in the smoking group were from 10 men ranging in age from 54 to 77 years old, and specimens in the non-smoking group were from 7 men and 8 women ranging in age from 49 to 89 years old. The normal oral mucosa from 6 women and 4 men (age range 24-54 years old) were used as control. The present study was approved by the Human Research Ethics Committee of Capital Medical University School of Stomatology and all subjects signed informed consents.

\section{HE and immunohistochemical staining}

Pathological diagnosis on tissue lesions was made based on HE staining according to the World Health Organization classification of head and neck tumors (4th Edition, 2017) (9). The histological changes in tongue mucosa and the degree of lesion were recorded as normal mucosa, hyperplasia, dysplasia, and OSCC. Immunohistochemistry (IHC) streptavidin-peroxidase two-step staining was performed to detect the expression of Prx1 binding proteins. After incubation in 3\% hydrogen peroxide at room temperature for $15 \mathrm{~min}$, the sections were incubated with primary antibodies to PPP2R1A (1:400), TPM3 (1:50), or CFL1 (1:400) (all from Abcam) overnight at $4^{\circ} \mathrm{C}$. Following incubation in secondary anti-rabbit IgG (Abcam) for $30 \mathrm{~min}$, slides were stained with chromogen diaminobenzidine (DAB) (Maixin, China) staining and hematoxylin redying. Three representative regions were selected at $200 \times$ magnification under the microscope Olympus BX61 (Olympus, Japan). Image Pro Plus software (Media Cybernetics, USA) was used for quantitative analyses and MOD (integral optical density (IOD)/measurement area) was calculated.

\section{Duolink in situ interaction assay in patient tissue specimens}

A pair of proximity ligation assay (PLA) probes targeting Prx1 and its interacting proteins were used in this assay. When the two PLA probes are in close proximity to each other, the oligonucleotide in PLA probes form a DNA circle template for the amplification with fluorescent signals. After being dewaxed and dehydrated with xylene and ethanol, tissues were permeabilized in PBS for $20 \mathrm{~min}$ at room temperature. Two probes (mouse- or rabbit-derived) with oligonucleotide-labeled secondary 
antibodies were added after incubation with primary antibodies against Prx1 (1:1000), CFL1 (1:200), TPM3 (1:200), and PPP2R1A (1:200). Blocking, staining, hybridization, ligation, amplification, and detection were performed in a humidity chamber. Fluorescence was visualized in the microscope Olympus BX61.

\section{Statistical analysis}

Differences were analyzed by one-way analysis of variance (SPSS v17.0, IBM, USA), and a P-value less than 0.05 was considered statistically significant.

\section{Results}

\section{Screening of Prx1 interacting proteins related to cell proliferation}

As analyzed by the Mascot database and UniProt database, 358 and 580 of candidate Prx1 interacting proteins were identified in the control and nicotine treatment groups, respectively. Through bioinformatics analysis, CFL1, TPM3, and PPP2R1A were screened out from the candidate Prx1 interacting proteins, which were related to cell proliferation (Tables 1 and 2).

Effect of nicotine on expression of CFL1, PPP2R1A, and TPM3 in induced OLK in Prx1 ${ }^{+/+}$mice

Histological observation and pathological diagnosis showed that all animals treated with either 4NQO or $4 \mathrm{NQO}+$ nicotine developed OLK with epithelial hyperplastic and dysplastic lesions in the tongue mucosa. As shown in Figure 1, we found that mice treated with $4 \mathrm{NQO}$ showed significantly higher expression of CFL1 than control animals $(\mathrm{P}<0.01)$ both in $\mathrm{Prx} 1^{+/+}$and $\mathrm{Prx} 1^{+/-}$ mice. Additionally, $4 \mathrm{NQO}+$ nicotine treatment promoted further expression of CFL1 compared to treatment with $4 \mathrm{NQO}$ alone in Prx1 ${ }^{+/+}$mice.

As shown in Figure 2, compared with the control, animals treated with $4 \mathrm{NQO}$ expressed a significantly higher level of PPP2R1A both in tongue tissues of Prx $1^{+/+}$and Prx $1^{+/-}$mice $(P<0.01)$. However, in Prx $1^{+/+}$mice, the PPP2R1A expression was significantly reduced by treating with $4 \mathrm{NQO}+$ nicotine compared to treating with $4 \mathrm{NQO}$ alone.

TPM3 was upregulated in 4NQO-induced OLK in both Prx $1^{+/+}$and Prx $1^{+/-}$mice (Figure 3, $\mathrm{P}<0.01$ ), but no significant change in TPM3 expression was detected comparing tongue tissues treated with $4 \mathrm{NQO}+$ nicotine to those treated with $4 \mathrm{NQO}$ in $\operatorname{Prx} 1^{+/+}$mice.

\section{CLF1, PPP2R1A, and TMP3 expression in induced}

OLK in Prx1 ${ }^{+/-}$mice treated with nicotine

As shown in Figures 1, 2, and 3, no significant changes in the expression of CFL1, PPP2R1A, and TPM3 were found in $4 \mathrm{NQO}+$ nicotine treatment of $\mathrm{Prx} 1^{+/-}$mice compared to $4 \mathrm{NQO}$ group.

\section{Verification of the Prx1 interacting proteins in human OLK tissues}

As shown in Figure 4, the expression of CFL1, PPP2R1A, and TPM3 significantly increased in OLK

Table 1. Spectrum identification of peroxiredoxin $1(\operatorname{Prx} 1)$ interacting proteins.

\begin{tabular}{|c|c|c|c|c|c|c|}
\hline Accession & Description & Gene name & $\mathrm{MW}[\mathrm{kDa}]$ & $\mathrm{PI}$ & Coverage & Unique peptides \\
\hline P23528 & Cofilin-1 & CFL1 & 18.5 & 8.09 & 43.98 & 6 \\
\hline P06753 & Tropomyosin alpha-3 chain & TPM3 & 32.9 & 4.72 & 22.81 & 1 \\
\hline P30153 & $\begin{array}{l}\text { Serine/threonine-protein phosphatase } 2 \mathrm{~A} 65 \mathrm{kDa} \\
\text { regulatory subunit } \mathrm{A} \text { alpha isoform }\end{array}$ & PPP2R1A & 65.3 & 5.11 & 3.90 & 2 \\
\hline
\end{tabular}

MW: molecular weight; PI: isoelectric point.

Table 2. Gene ontology (GO) analysis of peroxiredoxin 1 (Prx1) interacting proteins.

\begin{tabular}{lcc}
\hline Gene name & GO number & GO term \\
\hline CFL1 & GO:0043066 & Negative regulation of apoptotic process \\
& GO:0022604 & Regulation of cell morphogenesis \\
GO:0030010 & Establishment of cell polarity \\
TPM3 & GO:0006928 & Cellular component movement \\
PPP2R1A & GO:0006915 & Apoptotic process \\
& GO:0030155 & Negative regulation of cell growth \\
& GO:0040008 & Regulation of cell adhesion \\
& GO:0000188 & Regulation of growth \\
& & Inactivation of MAPK activity \\
\hline
\end{tabular}



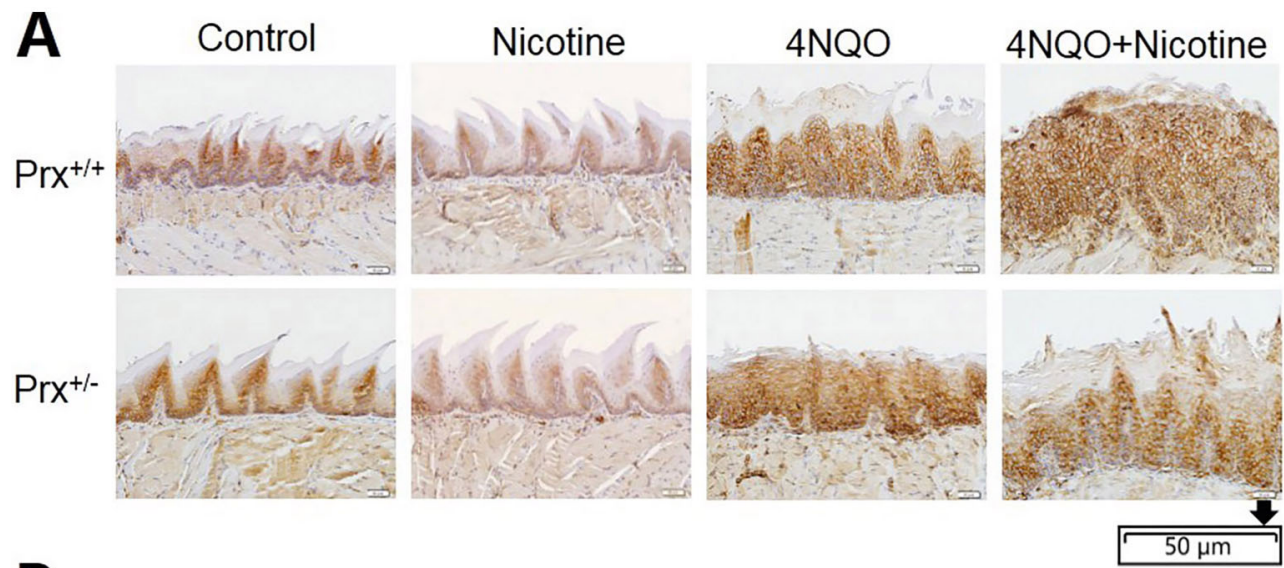

B

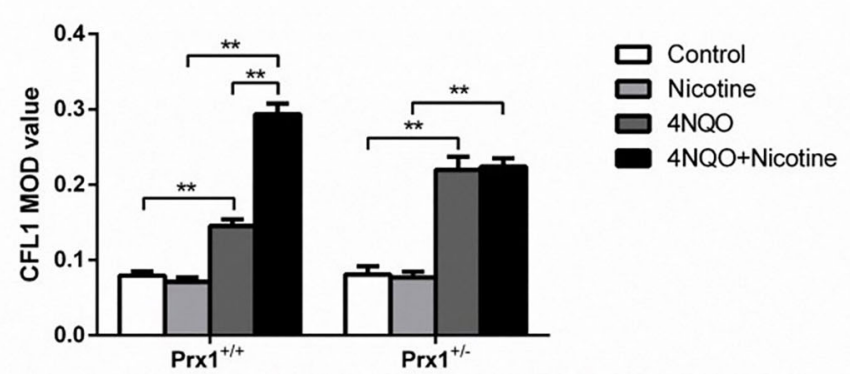

Figure 1. Immunohistochemical analysis of CFL1 in the tongue oral leukoplakia tissues of Prx $1^{+/+}$and Prx $1^{+/-}$mice. A, Representative immunohistochemistry images of CFL1 $(\times 200$, scale bar $50 \mu \mathrm{m})$. B, The integral optical density (IOD)/measurement area (MOD) values are reported as means $\pm \mathrm{SE}$. ${ }^{* *} \mathrm{P}<0.01$ (ANOVA). 4NQO: 4-nitro-quinoline-1-oxide.

compared to normal tissues. When comparing OLK of smokers and non-smokers, the expression level of CFL1 was higher $(P<0.05)$, while the expression of PPP2R1A was lower $(P<0.01)$. However, no significant difference in TPM3 expression was detected.

We then used an in situ Duolink assay to detect 3 candidate binding proteins in the above human OLK tissues of smokers. Positive red spots were found in these tissues, which confirmed that there are interactions of PPP2R1A, CFL1, and TPM3 with Prx1 in human OLK tissues (Figure 5). These results revealed a novel role of Prx1 involved in OLK development by interacting with CFL1, PPP2R1A, and TPM3.

\section{Discussion}

Cell proliferation is a vital indicator for understanding the mechanisms of function of certain genes, proteins, and pathways involved in cell survival and death. The cytoskeleton may induce cell proliferation through modulating cell hardness, and mechanical forces in cells can influence the cytoskeleton assembly, which in turn affects cell proliferation. In this study, three Prx1 interacting proteins, CFL1, PPP2R1A, and TPM3, related to cell proliferation, were screened out by mass spectrometry followed with GO and KEGG pathway analysis. According to existing reports, CFL1, PPPR1A, and TPM3 are all regulatory proteins in the dynamics of actin filaments. To confirm the binding of CFL1, PPPR1A, and TPM3 to Prx1, Duolink assay was conducted in human OLK tissues. Duolink analysis is used to detect the interaction between two proteins with high sensitivity and specificity in situ. The results can be interpreted with countable spots, each representing a single-molecule event. Duolink technique provides the benefits of traditional Co-IP plus the information of localization and the ability to study transient, weak interactions. We observed the interaction of CFL1, PPPR1A, and TPM3 with Prx1, which indicated that Prx1 performs the important function through its interaction network in OLK development; the bindings of CFL1 and PPPR1A with Prx1 were especially noteworthy in tobaccoassociated OLK.

Some preliminary studies of CFL1 and PPP2R1A involved in cell proliferation have been reported. In endometriotic patients, the phosphorylation of CFL1 regulates cell proliferation through the LIMK1/cofilin1 pathway (10). In eutopic endometrium of endometriosis patients, silencing CFL1 could block PDGF-induced proliferation (11). According to reports, expression of CFL1 varies in carcinomas including prostate, breast, lung, colorectal, and oral cancers, and the expression of CFL1 was increased in bladder cancer tissues compared 


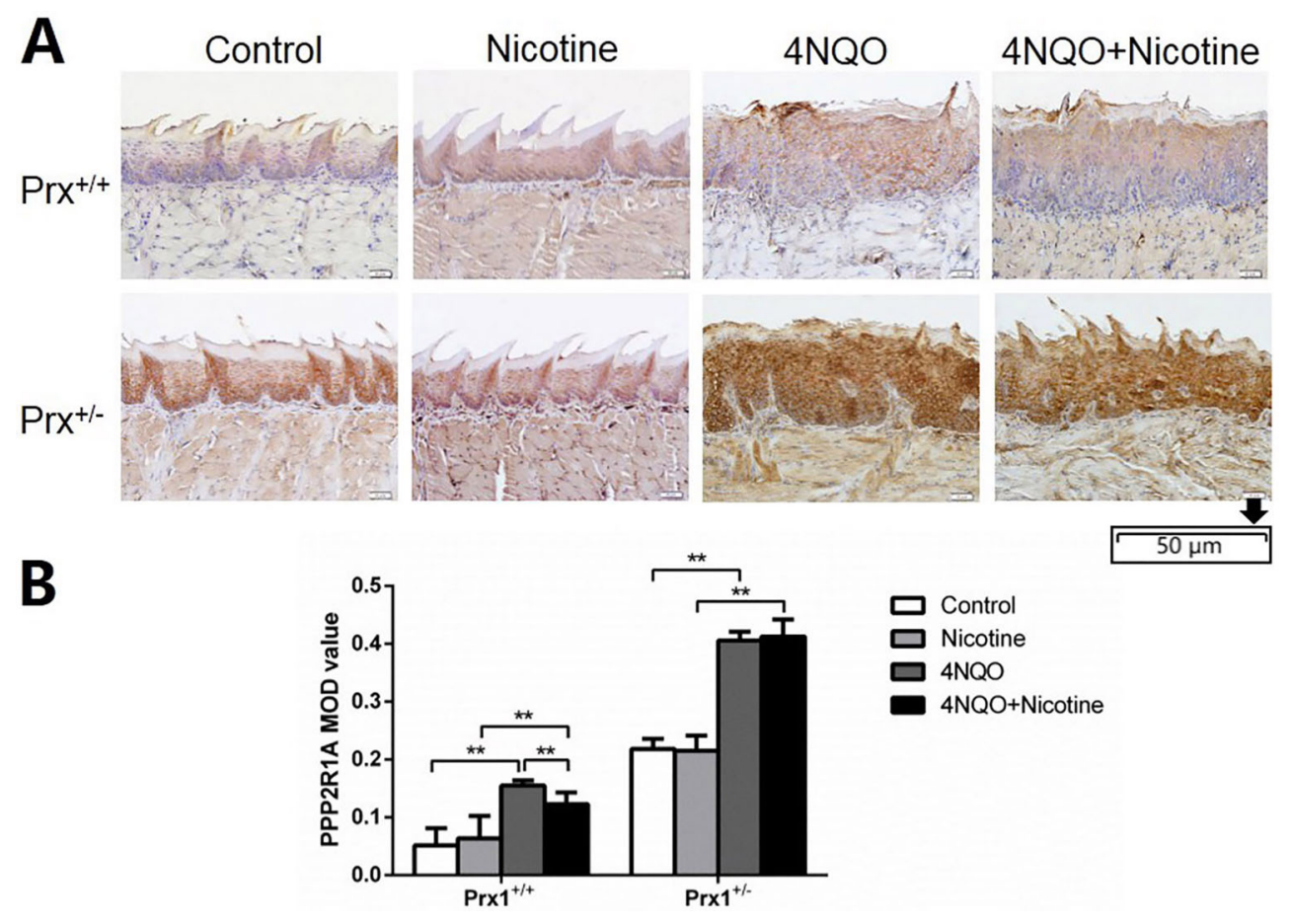

Figure 2. Immunohistochemical analysis of PPP2R1A in the tongue oral leukoplakia tissues of $\operatorname{Prx} 1^{+/+}$and $\operatorname{Prx} 1^{+/-}$mice. A, Representative immunohistochemistry images of PPP2R1A ( $\times 200$, scale bar $50 \mu \mathrm{m})$. B, The integral optical density (IOD)/ measurement area (MOD) values are reported as means $\pm \mathrm{SE} .{ }^{* *} \mathrm{P}<0.01$ (ANOVA). 4NQO: 4-nitro-quinoline-1-oxide.

with the precancerous lesion (12-14). Activated CFL1 participates in some essential biological processes of malignant tumors including proliferation, apoptosis, invasion, and chemo-resistance (15). PPP2R1A, an isoform of $\mathrm{PP} 2 \mathrm{~A}$, is an important serine/threonine protein phosphatase and is considered to be a tumor suppressor in some human malignancies (16). The mutations in PPP2R1A have been identified in multiple cancers, but the effects of these mutations on PP2A function need to be fully elucidated. Mutations of PPP2R1A promote cells proliferation by upregulating the phosphorylation levels of Akt, ERK, and WNK1 in gastrointestinal stromal tumors and by interactions with the PP2A inhibitor TIPRL1 in uterine cancer $(17,18)$. In alveolar rhabdomyosarcoma, silencing of PPP2R1A significantly increased cell growth, suggesting the tumor-suppressive function of PPP2R1A (19). Our results showed that the expression of CFL1 and PPP2R1A was higher in human and mouse OLK tissues than that in normal tissues, indicating their roles in the development of oral precancerous lesions. In addition, nicotine and Prx1 were associated with an upregulation of CFL1 and a downregulation of PPP2R1A in OLK. Notably, in $\operatorname{Prx} 1^{+/-}$mice, this association between nicotine and CFL1 or PPP2R1A was attenuated in OLK tissues, further highlighting the importance of the Prx1 interaction network in OLK. The results suggested that nicotine upregulated CFL1 and downregulated PPP2R1A both depending on
Prx1 and thus promoted the development of OLK. CFL1 or PPP2R1A could be a marker for the treatment of tobaccorelated OLK.

We also observed a higher expression of TPM3 in human and mouse OLK tissues in this study. TPM3 belongs to the tropomyosin super family, which can be viewed as a universal regulator of the actin cytoskeleton for cellular functions such as cell proliferation (20). The expression levels of TPM3 are higher in stage III esophageal squamous cell carcinoma tissue compared with stage I (21), and glucose glycated TPM3 suppresses colon cancer cell Caco-2 proliferation (22). In immortalized mouse embryonic fibroblasts, TPM3 is partly responsible for cell proliferation via regulating the interaction between pERK and Imp7, which is overridden by Ras transformation (23). Transfection of TPM3-anaplastic Iymphoma kinase $(A L K)$ fusion gene results in the loss of contact inhibition in NIH3T3 cells (24). The results of the current study suggested that TPM3 may be an important partner of the Prx1 network in regulating cell proliferation in OLK. However, nicotine had no significant effect on expression of TPM3 in 4NQO-induced OLK tissues. Further studies are needed to explore the mechanism of Prx1/TPM3 in tobacco-related OLK.

In this study, for the first time, we found that nicotine increased expression of CFL1 and decreased expression of PPP2R1A in 4NQO-induced OLK tissues by regulating 


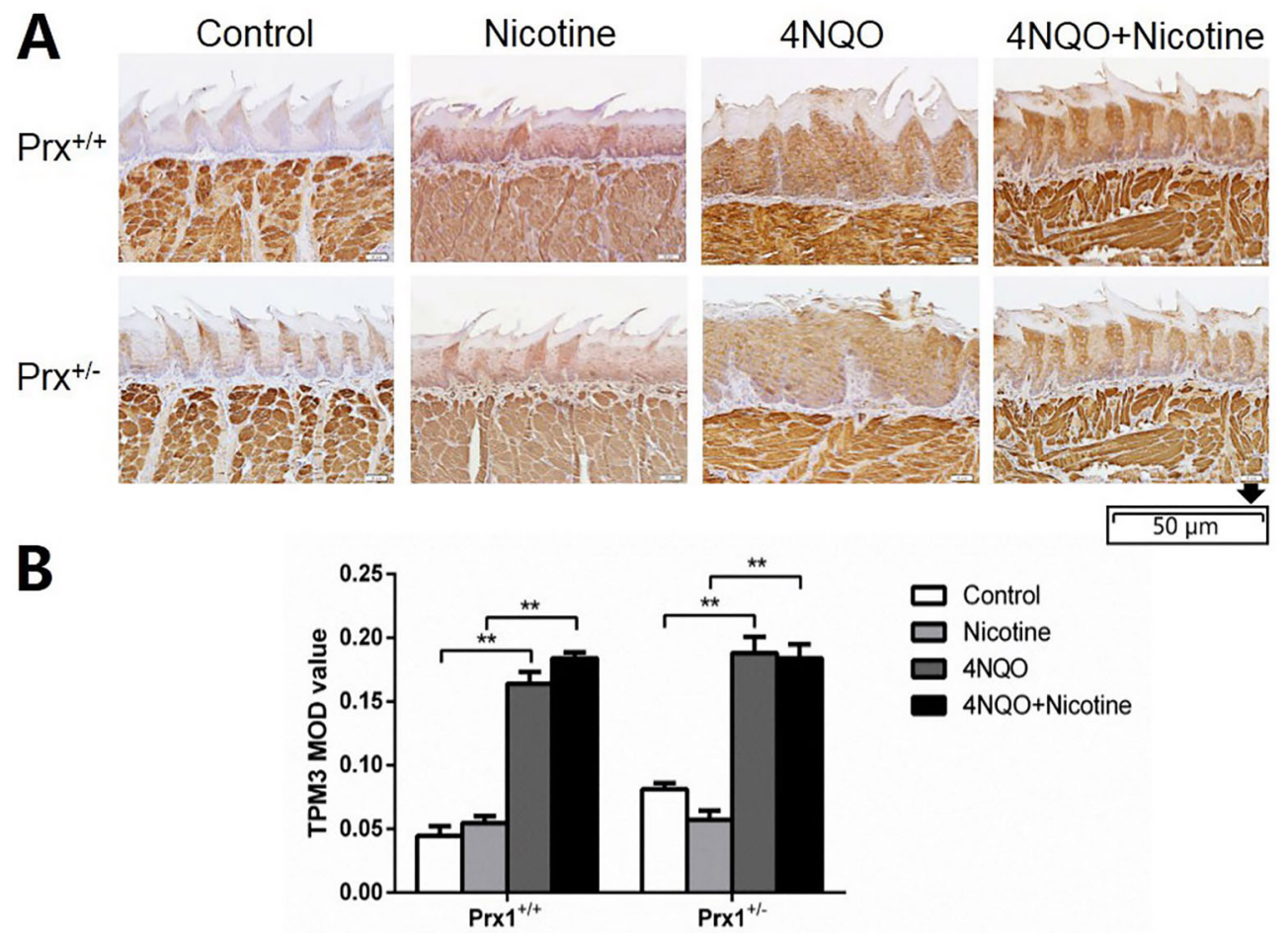

Figure 3. Immunohistochemical analysis of TPM3 in the tongue oral leukoplakia tissues of $\operatorname{Prx} 1^{+/+}$and $\operatorname{Prx} 1^{+/-}$mice. A, Representative images of TPM3 $(\times 200$, scale bar $50 \mu \mathrm{m})$. B. The integral optical density (IOD)/measurement area (MOD) values are reported as means $\pm \mathrm{SE}$. ${ }^{* *} \mathrm{P}<0.01$ (ANOVA). 4NQO: 4-nitro-quinoline-1-oxide.
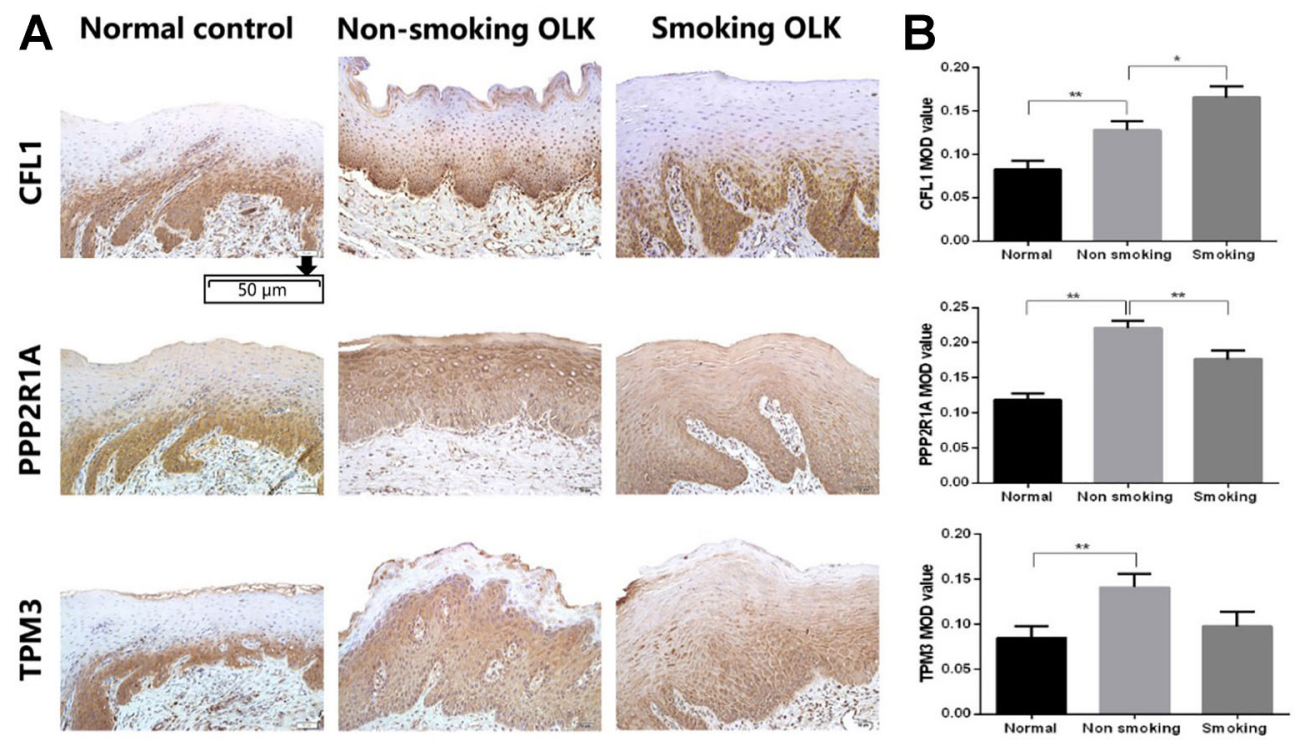

Figure 4. Immunohistochemical analysis of CFL1, PPP2R1, and TPM3 in human normal mucosa and oral leukoplakia (OLK) tissues of smokers and non-smokers. A, Representative images of PPP2R1, TPM3, and CFL1 in normal mucosa and OLK tissues ( $\times 200$, scale bar $50 \mu \mathrm{m})$. B, The integral optical density (IOD)/measurement area (MOD) values are reported as means $\pm S E$. ${ }^{*} P<0.05 ;{ }^{* *} P<0.01$ (ANOVA). 4NQO: 4-nitro-quinoline-1-oxide. 


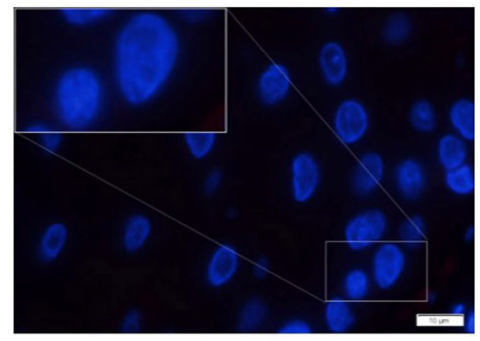

Control

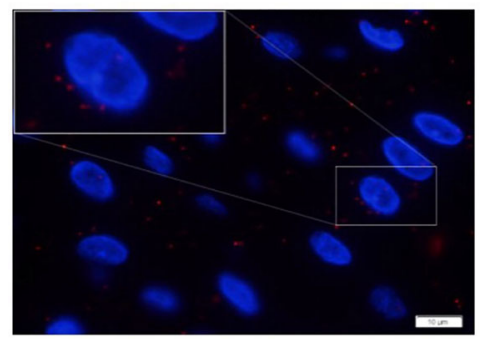

PPP2R1A

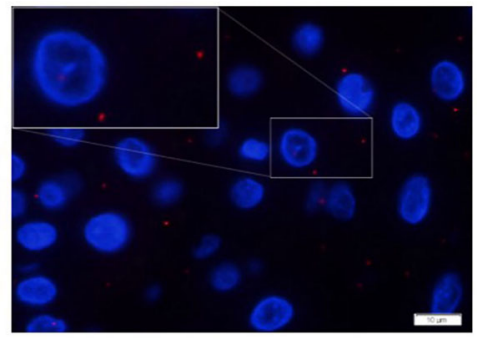

CFL1

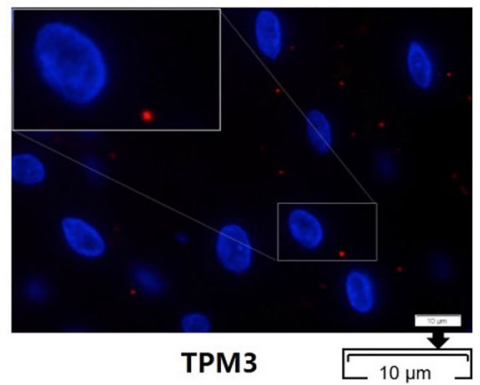

Figure 5. Duolink analysis. The interactions of PPP2R1A, TPM3, and CFL1 with Prx1 in human oral leukoplakia tissues were detected by in situ Duolink analysis. Proximity ligation assay signals are shown in red and the nuclei are stained in blue $(\times 1000$, scale bar $10 \mu \mathrm{m})$.

Prx1 in mice. The binding of CFL1, PPP2R1A, and TPM3 to Prx1 was confirmed in human OLK tissues. It suggested that tobacco promoted the development of OLK via regulating Prx1 and its interacting proteins CFL1 and PPP2R1A. Results of this study may be helpful to develop strategies for OLK prevention targeting Prx1 and its interacting proteins.

\section{References}

1. Narayan TV, Shilpashree S. Meta-analysis on clinicopathologic risk factors of leukoplakias undergoing malignant transformation. J Oral Maxilloface Pathol 2016; 20: 354361, doi: 10.4103/0973-029X.190900.

2. Choudhari SK, Chaudhary M, Gadbail AR, Sharma A, Tekade S. Oxidative and antioxidative mechanisms in oral cancer and precancer: a review. Oral Oncol 2014; 50: 10 18, doi: 10.1016/j.oraloncology.2013.09.011.

3. Grando SA. Connections of nicotine to cancer. Nat Rev Cancer 2014; 14: 419-429, doi: 10.1038/nrc3725.

4. Nicolussi A, D'Inzeo S, Capalbo C, Giannini G, Coppa A. The role of peroxiredoxins in cancer. Mol Clin Oncol 2017; 6: 139-153, doi: 10.3892/mco.2017.1129.

5. Karplus PA. A primer on peroxiredoxin biochemistry. Free Radic Biol Med 2015; 80: 183-190, doi: 10.1016/j. freeradbiomed.2014.10.009.

6. Ding C, Fan X, Wu G. Peroxiredoxin 1 - an antioxidant enzyme in cancer. J Cell Mol Med 2017; 21: 193-202, doi: $10.1111 / \mathrm{jcmm} .12955$.

7. Niu W, Zhang $M$, Chen $H$, Wang $C$, Shi $N$, Jing $X$, et al. Peroxiredoxin 1 promotes invason and migration by regulating epithelial-to-mesenchymal transition during oral

\section{Acknowledgments}

This study was funded by the National Natural Science Foundation of China (81470752) and the Scientific Research Common Program of Beijing Municipal Commission of Education (KM201910025009).

carcinogenesis. Oncotarget 2016; 7: 47042-47051, doi: 10.18632/oncotarget.9705.

8. Zhang J, Jing X, Niu W, Zhang M, Ge L, Miao C, et al. Peroxiredoxin 1 has an anti-apoptotic role via apoptosis signal-regulating kinase 1 and p38 activation in mouse models with oral precancerous lesions. Oncol Lett 2016; 12: 413-420, doi: 10.3892/ol.2016.4659.

9. Adel K El-Naggar, John KC Chan, Jennifer R Grandis, Takashi Takata, Pieter J Slootweg. World Health Organization classification of head and neck tumors.4th Edition. Lyon: IARC; 2017.

10. Liu J, Zhang Z, Liu J, Wang D. LIM kinase 1 mediates estradiol effects on the phosphorylation of cofilin1 in eutopic endometrial stromal cells during the invasion and proliferation of endometriosis. Reprod Sci 2019; 26: 1499-1505, doi: 10.1177/1933719119828076.

11. Wang DB, Xu YL, Chen P, Chen YH, Li Y. Silencing Cofilin-1 blocks PDGF-induced proliferation in eutopic endometrium of endometriosis patients. Cell Biol Int 2013; 37: 799-804, doi: 10.1002/cbin.10093.

12. Shishkin S, Eremina L, Pashintseva N, Kovalev L, Kovaleva M. Cofilin-1 and other ADF/cofilin superfamily members in 
human malignant cells. Int J Mol Sci 2016; 18: 10, doi: 10.3390/ijms18010010.

13. Wang F, Wu D, Fu H, He F, Xu C, Zhou J, et al. Cofilin 1 promotes bladder cancer and is regulated by TCF7L2. Oncotarget 2017; 8: 92043-92054, doi: 10.18632/oncotarget. 20664.

14. Chanthammachat $\mathrm{P}$, Promwikorn $\mathrm{W}$, Pruegsanusak K, Roytrakul S, Srisomsap C, Chokchaichamnankit D, et al. Comparative proteomic analysis of oral squamous cell carcinoma and adjacent non-tumour tissue from Thailand. Arch Oral Biol 2013; 58: 1677-1685, doi: 10.1016/j. archoralbio.2013.08.002.

15. Liu W, Zhang Q, Tang Q, Hu C, Huang J, Liu Y, et al. Lycorine inhibits cell proliferation and migration by inhibiting ROCK1/cofilin-induced actin dynamics in HepG2 hepatoblastoma cells. Oncol Rep 2018; 40: 2298-2306, doi: 10.3892/or.2018.6609.

16. Seshacharyulu P, Pandey P, Datta K, Batra SK. Phosphatase: PP2A structural importance, regulation and its aberrant expression in cancer. Cancer Lett 2013; 335: 9-18, doi: 10.1016/j.canlet.2013.02.036.

17. Toda-Ishii M, Akaike K, Suehara Y, Mukaihara K, Kubota D, Kohsaka $S$, et al. Clinicopathological effects of protein phosphatase 2, regulatory subunit $A$, alpha mutations in gastrointestinal stromal tumors. Mod Pathol 2016; 29: 14241432, doi: 10.1038/modpathol.2016.138.

18. Haesen $D$, Abbasi Asbagh L, Derua R, Hubert A, Schrauwen $S$, Hoorne $\mathrm{Y}$, et al. Recurrent PPP2R1A mutations in uterine cancer act through a dominant-negative mechanism to promote malignant cell growth. Cancer Res 2016; 76: 5719-5731, doi: 10.1158/0008-5472.CAN-15-3342.

19. Akaike K, Suehara $\mathrm{Y}$, Kohsaka S, Hayashi T, Tanabe $\mathrm{Y}$, Kazuno $\mathrm{S}$, et al. PPP2R1A regulated by PAX3/FOXO1 fusion contributes to the acquisition of aggressive behavior in PAX3/FOXO1-positive alveolar rhabdomyosarcoma. Oncotarget 2018; 9: 25206-25215, doi: 10.18632/oncotarget. 25392.

20. Hitchcock-DeGregori SE, Barua B. Tropomyos in structure, function, and interactions: a dynamic regulator. Subcell Biochem 2017; 82: 253-284, doi: 10.1007/978-3-319-49674-0.

21. Yu SB, Gao Q, Lin WW, Kang MQ. Proteomic analysis indicates the importance of TPM3 in esophageal squamous cell carcinoma invasion and metastasis. Mol Med Rep 2017; 15: 1236-1242, doi: 10.3892/mmr.2017.6145.

22. Zhang $Z$, Xiao $H$, Zhang $X$, Zhou P. Conformation, allergenicity and human cell allergy sensitization of tropomyosin from Exopalaemon modestus: effects of deglycosylation and Maillard reaction. Food Chem 2019; 276: 520527, doi: 10.1016/j.foodchem.2018.10.032.

23. Coombes JD, Schevzov G, Kan CY, Petti C, Maritz MF, Whittaker $S$, et al. Ras transformation overrides a proliferation defect induced by Tpm3.1 knockout. Cell Mol Biol Lett 2015; 20: 626-646, doi: 10.1515/cmble-2015-0037.

24. Amano $\mathrm{Y}$, Ishikawa $\mathrm{R}$, Sakatani $\mathrm{T}$, Ichinose J, Sunohara $\mathrm{M}$, Watanabe K, et al. Oncogenic TPM3-ALK activation requires dimerization through the coiled-coilstructure of TPM3. Biochem Biophys Res Commun 2015; 457: 457460, doi: 10.1016/j.bbrc.2015.01.014. 